\title{
Four Variables Suffice
}

\author{
Alasdair Urquhart* \\ Department of Philosophy \\ University OF TORONTO \\ urquhart@cs.toronto.edu \\ Received by Greg Restall \\ Published November I0, 2007 \\ http://www .philosophy . unimelb.edu.au/ajl/2007 \\ (C) 2007 Alasdair Urquhart
}

\section{INTRODUCTION}

In one of his wittiest and most incisive papers [3], Robert K. Meyer has described Belnap's attack on the decision problems for the principal relevant propositional logics as an inductive procedure. Belnap began from the decidability of the first degree fragment of these logics, and proved the decidability of successively more complicated fragments, where the complexity was measured by the degree of nesting of entailments. Meyer proved the amazing result that it was already sufficient to prove the decidability of the second degree fragment of these logics. What is even more amazing, the proof is completely straightforward, depending on nothing more than the introduction of new variables as abbreviations for complex formulas, and replacement of equivalent propositions in the logics in question. In retrospect, we can recognize that this technique of abbreviations is the same as that used by Tseitin in his pioneering paper of 1968 on the complexity of propositional proofs [7], but Meyer seems to have been the first to recognize the general applicability of the technique in logics other than classical propositional calculus. Now that we know that these logics are undecidable, it follows at once that their second degree fragments are undecidable.

What I wish to propose in the present paper is a new form of "career induction" for ambitious young logicians. In this case, the induction proceeds downward rather than upward. The basic problem is this: if we look at the $n$-variable fragments of these propositional logics, at what point does undecidability begin? Let's focus, to be definite, on the logic R. John Slaney [5]

\footnotetext{
*The author gratefully acknowledges the support of the Natural Sciences and Engineering Research Council of Canada.
} 
showed that the 0-variable fragment of $\mathbf{R}$ (where we allow the sentential constants $t$ and $\mathrm{f}$ ) contains exactly 3088 non-equivalent propositions, and so is clearly decidable. In the opposite direction, I claimed in my paper of 1984 that the five variable fragment of $\mathbf{R}$ is undecidable. The proof given there was sketchy (to put the matter charitably), and a close examination reveals that although the result claimed is true, the proof given is incorrect (something that escaped even the eagle eye of the Maximum Leader of the Logicians Liberation League). In the present paper, I give a detailed and (I hope) correct proof that the four variable fragments of the principal relevant logics are undecidable. This leaves open the question of the decidability of the $n$-variable fragments for $n=1,2,3$. At what point does undecidability set in?

\section{COORDINATE FRAMES IN ALGEBRAS}

In this section, we outline the definition of multiplication in a coordinate frame that is used to encode the equations constituting the presentation of a semigroup with undecidable word problem. We follow the exposition of [8] (see also [I, \$65.2]).

An algebra $\langle S ; \wedge,+, \cup, \perp, 0\rangle$ is defined to be a t-monoid if it satisfies the following conditions:

I. $\langle S ; \wedge, \perp\rangle$ is a meet semilattice with least element $\perp$, where $\leqslant$ is defined as $a \wedge b=a$,

2. $\langle S ;+, 0\rangle$ forms a commutative monoid,

3. $0 \leqslant y \Rightarrow x \leqslant x+y$,

4. $x \leqslant y, z \leqslant w \Rightarrow x+z \leqslant y+w, x \cup z \leqslant y \cup w$,

5. $x+(y \cup z)=(x+y) \cup(x+z)$,

6. $x \cup \perp=x=\perp \cup x$.

We shall use juxtaposition for the meet operation to reduce the apparent complexity of terms. We use $\Sigma X$ for $x_{1}+\cdots+x_{n}$, where $X=\left\{x_{1}, \ldots, x_{n}\right\}$. We define an element $a$ in a $t$-monoid to be modular if it satisfies the condition:

$$
\forall \mathrm{b} \forall \mathrm{c}(\mathrm{a} \geqslant \mathrm{c} \Rightarrow \mathrm{a}(\mathrm{b}+\mathrm{c})=\mathrm{ab}+\mathrm{c}) .
$$

Let $M$ be a $t$-monoid. A family of elements $\left\{a_{1}, \ldots, a_{n}\right\} \cup\left\{c_{i j}: i \neq j, 1 \leqslant\right.$ $i, j \leqslant n\}$ is an $n$-frame if it satisfies the conditions:

I. For $G, H \subseteq\left\{a_{1}, \ldots, a_{n}\right\},(\Sigma G)(\Sigma H)=\Sigma(G \cap H)$, where $\Sigma \emptyset=0$;

2. $a_{i}+a_{i}=a_{i}$;

3. $\left(c_{i j}+c_{j k}\right)\left(a_{i}+a_{k}\right)=c_{i k}$, where $i, j, k$ are distinct; 
4. $c_{i j}=c_{j i}$

5. $c_{i j}+a_{j}=a_{i}+a_{j}$ and $c_{i j} a_{j}=a_{j}$.

An $n$-frame is said to be modular if $\Sigma G$ is modular for $G \subseteq\left\{a_{1}, \ldots, a_{n}\right\}$.

The first three parts of the definition say that $a_{1}, \ldots, a_{n}$, provided they are distinct, generate a copy of the Boolean algebra $2^{n}$ with 0 as the lattice zero and the $a_{i}$ as atoms (in von Neumann's terminology, the set $\left\{a_{1}, \ldots, a_{n}\right\}$ is independent). The elements $c_{i j}$ serve as centres of perspectivity with respect to the coordinate frame.

For the remainder of this section we assume that we are dealing with a fixed $t$-monoid $M$ containing a modular $n$-frame with $n \geqslant 4$; subscripts $i, j, k$ etc. are assumed to range over $1, \ldots, n$.

For distinct $i, j$, we define:

$$
\mathrm{L}_{i j}=\left\{x \in M \mid x+a_{j}=a_{i}+a_{j}, x \wedge a_{j}=0\right\},
$$

and for distinct $i, j, k, b \in L_{i j}, d \in L_{j k}$,

$$
b \otimes d=(b+d)\left(a_{i}+a_{j}\right),
$$

and for $x, y \in \mathrm{L}_{12}$,

$$
x \odot y=\left(x \otimes c_{23}\right) \otimes\left(c_{31} \otimes y\right) .
$$

It is possible to show that in a t-monoid, the multiplication $x \odot y$ defined relative to a modular $n$-frame, with $n \geqslant 4$, is associative. For details, see $[8]$ and [I, \$65.2].

In order to cut down on the number of variables needed for undecidability, we need to prove lemmas showing that in certain $t$-monoids, an $n$-frame and given elements $r, s$ of $L_{12}$ in the frame can be generated algebraically from a smaller set of elements. First, we prove four lemmas that hold in general $t$-monoids. The elements $c_{i j}, a_{k}$ in the lemmas below are assumed to be elements of an $n$-frame in a $t$-monoid.

Lemma I For any $i, j, k, c_{i j} \wedge a_{k}=0$.

Proof: If $k \notin\{i, j\}$, then since $c_{i j} \leqslant a_{i}+a_{j}, c_{i j} \wedge a_{k} \leqslant\left(a_{i}+a_{j}\right) a_{k}=0$. If $k=j$, then since $c_{i j} \in L_{i j}, c_{i j} \wedge a_{k}=0$. Similarly, if $k=i, c_{i j} \wedge a_{k}=c_{j i} \wedge a_{i}=0$.

LEMMA 2 If $x \in \mathrm{L}_{i j}$, and $\{i, j\} \neq\{k, l\}$, then $x \wedge c_{k l}=0$.

Proof: Let us suppose that $l \notin\{i, j\}$. Since $x \in L_{i j}, x \leqslant a_{i}+a_{j}$, and since $c_{k l} \in L_{k l}, c_{k l} \leqslant a_{k}+a_{l}$, so that $x \wedge c_{k l} \leqslant\left(a_{i}+a_{j}\right)\left(a_{k}+a_{l}\right)$. If $\{i, j\} \cap\{k, l\}=\emptyset$, then $\left(a_{i}+a_{j}\right)\left(a_{k}+a_{l}\right)=0$. So let us suppose that $i=k$. Then $x \wedge c_{k l} \leqslant\left(a_{i}+\right.$ $\left.a_{j}\right)\left(a_{k}+a_{l}\right)=a_{i}=a_{k}$. Hence by Lemma元, $x \wedge c_{k l} \leqslant x \wedge c_{k l} \wedge a_{i} \leqslant x \wedge 0=0$.

LEMMA 3 Let $\mathrm{r}, \mathrm{s} \in \mathrm{L}_{\mathrm{ij}}$, where $\mathrm{r} \leqslant \mathrm{s}$ and $\mathrm{s}$ is modular. Then $\mathrm{r}=\mathrm{s}$. 
Proof: Since $s$ is modular, $s \leqslant s\left(a_{i}+a_{j}\right)=s\left(r+a_{j}\right)=s a_{j}+r=0+r=r$.

Lemma 4 Assume that $\mathrm{s} \in \mathrm{L}_{12}$, and that $\mathrm{s}+\mathrm{c}_{23}$ and $\left(\mathrm{s} \otimes \mathrm{c}_{23}\right) \otimes \mathrm{c}_{23}$ are modular. Then $\mathrm{s}=\left(\mathrm{s} \otimes \mathrm{c}_{23}\right) \otimes \mathrm{c}_{23}$.

Proof: Since $s+c_{23}$ is modular, we have

$$
\begin{aligned}
\left(s \otimes c_{23}\right)+c_{23} & =\left(s+c_{23}\right)\left(a_{1}+a_{3}\right)+c_{23} \\
& =\left(s+c_{23}\right)\left(a_{1}+a_{3}+c_{23}\right) \\
& =\left(s+c_{23}\right)\left(a_{1}+a_{2}+a_{3}\right) .
\end{aligned}
$$

Hence, $s \leqslant\left(\left(s \otimes c_{23}\right)+c_{23}\right)\left(a_{1}+a_{2}\right)=\left(s \otimes c_{23}\right) \otimes c_{23}$. By Lemma 2.5 of $[8]$, $\left(s \otimes c_{23}\right) \otimes c_{23} \in L_{12}$, so by Lemma 3, $s=\left(s \otimes c_{23}\right) \otimes c_{23}$.

The t-monoid in the next lemma is a Boolean t-monoid. By this we mean a $\mathrm{t}$-monoid in which we have an additional one-place operation $\neg$ defined on the universe $S$ so that $\langle S ; \wedge, \neg, \cup, \perp\rangle$ forms a Boolean algebra, with least element $\perp$, complement operation $\neg$, and $\wedge$ and $\cup$ the meet and union operations.

Lemma 5 Let $M$ be a Boolean t-monoid, and $\left\{a_{1}, \ldots, a_{4}\right\} \cup\left\{c_{i j}: i \neq j, 1 \leqslant i, j \leqslant 4\right\}$ a 4-frame in $\mathrm{M}$. Let $\mathrm{r} \in \mathrm{L}_{12}, \mathrm{u} \in \mathrm{L}_{13}$ and define: $\mathrm{X}=\mathrm{r} \cup \mathrm{a}_{3}, \mathrm{Y}=\mathrm{u} \cup \mathrm{a}_{2}$ and $Z=c_{14} \cup c_{24} \cup c_{34} \cup a_{2} \cup a_{3}$. Then all of the elements of the frame, together with $r$ and $\mathrm{u}$, are in the subalgebra generated by the elements $\mathrm{X}, \mathrm{Y}, \mathrm{Z}, \mathrm{a}_{4}$.

Proof: First, we show that $X \wedge Z=a_{3}$. We have by the distributive law and Lemma2,

$$
\begin{aligned}
r \wedge Z & =r \wedge\left(c_{14} \cup c_{24} \cup c_{34} \cup a_{2} \cup a_{3}\right) \\
& =\left[r \wedge\left(c_{14} \cup c_{24} \cup c_{34}\right)\right] \cup\left[r \wedge\left(a_{2} \cup a_{3}\right)\right] \\
& =0 \cup\left(r \wedge a_{2}\right) \cup\left(r \wedge a_{3}\right) \\
& =0
\end{aligned}
$$

since $r \wedge a_{3} \leqslant\left(a_{1}+a_{2}\right) a_{3}=0$. Again, by distributivity and Lemma $\mathrm{I}$, we have:

$$
\begin{aligned}
a_{3} \wedge z & =a_{3} \wedge\left(c_{14} \cup c_{24} \cup c_{34} \cup a_{2} \cup a_{3}\right) \\
& =0 \cup a_{3} \\
& =a_{3} .
\end{aligned}
$$

Hence, $X \wedge Z=0 \cup a_{3}=a_{3}$. By a symmetrical argument, $Y \wedge Z=a_{2}$; from this we also have $0=(X \wedge Y \wedge Z)$.

Now we have (by the properties of the Boolean complement):

$$
\begin{aligned}
\mathrm{x} \wedge \neg \mathrm{a}_{3} & =\left(\mathrm{r} \cup \mathrm{a}_{3}\right) \wedge \neg \mathrm{a}_{3} \\
& =\left(\mathrm{r} \wedge \neg \mathrm{a}_{3}\right) \cup\left(\mathrm{a}_{3} \wedge \neg \mathrm{a}_{3}\right) \\
& =\mathrm{r} \wedge \neg \mathrm{a}_{3} .
\end{aligned}
$$


Since $r \in L_{12}, r \leqslant a_{1}+a_{2}$, so $r \wedge a_{3} \leqslant\left(a_{1}+a_{2}\right) a_{3}=0$, hence

$$
\begin{aligned}
r & =\left(r \wedge a_{3}\right) \cup\left(r \wedge \neg a_{3}\right) \\
& =0 \cup\left(r \wedge \neg a_{3}\right) \\
& =0 \cup\left(X \wedge \neg a_{3}\right) .
\end{aligned}
$$

Similarly, $u=\left(Y \wedge \neg a_{2}\right) \cup 0$. Since $r \in L_{12}, u \in L_{13}, r+a_{2}=a_{1}+a_{2}$, $u+a_{3}=a_{1}+a_{3}$, hence $a_{1}=\left(a_{1}+a_{2}\right)\left(a_{1}+a_{3}\right)=\left(r+a_{2}\right)\left(u+a_{3}\right)$.

It remains to show that the elements $c_{i j}$ are also in the subalgebra generated by $X, Y, Z, a_{4}$. We have $c_{24}\left(a_{1}+a_{4}\right) \leqslant c_{24}\left(a_{2}+a_{4}\right)\left(a_{1}+a_{4}\right) \leqslant c_{24} a_{4}=0$; similarly, $c_{34}\left(a_{1}+a_{4}\right) \leqslant 0$. Hence, by distribution, $Z \wedge\left(a_{1}+a_{4}\right)=c_{14}$, and similarly $Z \wedge\left(a_{2}+a_{4}\right)=c_{24}, Z \wedge\left(a_{3}+a_{4}\right)=c_{34}$. The remaining elements $c_{i j}$ can be generated by the equations $\left(c_{i j}+c_{j k}\right)\left(a_{i}+a_{k}\right)=c_{i k}$, where $i, j, k$ are distinct.

\section{UNDECIDABILITY IN FOUR VARIABLES}

In this section, we apply the algebraic lemmas of the previous section to show the undecidability of the four variable fragment of various relevant logics. We assume that we are working in a formulation of relevant logics that includes negation, but not the propositional constants $t$ and $T$.

Let us recall the construction of Urquhart [8] that proves undecidability for $\mathbf{R}$ and other logics. We are given a finitely presented semigroup $\mathrm{P}$ with undecidable word problem; we can assume that the presentation of $\mathrm{P}$ consists of a finite set of equations $\phi_{1}=\psi_{1}, \ldots, \phi_{n}=\psi_{n}$ in two variables $r$, s. The argument of [8, Theorem 5.I] shows that there is a way of translating these equations into $\mathbf{R}$ so that a given equation $\epsilon=\delta$ is deducible from the presentation if and only its translation $\epsilon^{t}=\delta^{t}$ is deducible from the translation of the presentation, together with an auxiliary set of formulas expressed in an additional sixteen variables $a_{1}, a_{2}, a_{3}, a_{4}, c_{12}, \ldots, c_{43}$, corresponding to the elements of a 4 -frame. Thus the argument, without any added effort, shows undecidability for the eighteen-variable fragment of these logics.

Let $A$ be a formula of $\mathbf{R}$ built up from these eighteen variables $r, s, a_{1}$, $a_{2}, a_{3}, a_{4}, c_{12}, \ldots, c_{43}$, together with the constants $t$ and $T$. Let us think of these variables as denoting the elements of a Boolean monoid, as in Lemma 5 in addition, assume that the conditions of Lemma 4 holds. Define $u=$ $\left(s \otimes c_{23}\right)$. Then Lemmas 4 and 5 show us how to express all of the elements corresponding to the eighteen variables, and also the constants $t$ and $T$, in terms of the elements $X, Y, Z, a_{4}$; for example, $a_{3}$ can be expressed as $X \wedge Z$. Let $A^{\sigma}$ be the result of replacing each of the variables in $A$, as well as the constants $t$ and $T$ by the expressions in the four variables $X, Y, Z, a_{4}$ that serve to define them in a Boolean $t$-monoid satisfying the conditions of Lemma 4

We have now enough material to prove the main result of this paper. Let $\mathbf{L}(\mathbf{V})$ be the logic determined by the model $M(V)$ constructed from an 
infinite-dimensional vector space (for details see [8, Lemma 4.4]). The systems $\mathbf{T}-\mathbf{W}$ and $\mathbf{T}-\mathbf{W}+\mathbf{A r 5}$ are the systems of ticket entailment minus contraction, and the same system with the added axiom scheme $((A \rightarrow B) \wedge A \wedge t) \rightarrow B$.

Theorem 6 I. If $\mathrm{L}$ is a logic intermediate between $\mathbf{T}-\mathbf{W}$ and $\mathbf{L}(\mathbf{V})$, then the deducibility problem for formulas in four variables in $\mathrm{L}$ is unsolvable.

2. If $\mathrm{L}$ is a logic intermediate between $\mathbf{T}-\mathbf{W}+\mathbf{A r} 5$ and $\mathbf{L}(\mathbf{V})$, then the deducibility problem for formulas in four variables in $\mathrm{L}$ is unsolvable.

Proof: Let $\Delta$ be the translation of the semigroup presentation into the language of $\mathbf{R}$, together with the auxiliary formulas, and let $\epsilon^{t}=\delta^{t}$ be the translation of a given semigroup equation $\epsilon=\delta$ in the variables $r$, s. We wish to show that $\epsilon=\delta$ is deducible from the semigroup presentation if and only if $\left(\epsilon^{t}=\delta^{t}\right)^{\sigma}$ is deducible from $\Delta^{\sigma}$.

The argument of $[8$, shows that if $\epsilon=\delta$ is deducible from the presentation, then $\epsilon^{t}=\delta^{t}$ is deducible from $\Delta$ in L. Hence, by substitution, $\left(\epsilon^{t}=\delta^{t}\right)^{\sigma}$ is deducible from $\Delta^{\sigma}$.

For the converse, if $\epsilon=\delta$ is not deducible from $\Delta$, then it is possible to interpret the variables $r, s, a_{1}, a_{2}, a_{3}, a_{4}, c_{12}, \ldots, c_{43}$ in the model $M(V)$ so that (I) the variables $a_{1}, a_{2}, a_{3}, a_{4}, c_{12}, \ldots, c_{43}$ denote a 4 -frame in the modular lattice of idempotents $\geqslant t$ in the model, (2) r, s denote elements of $L_{12}$ in this 4 -frame, (3) the interpretation of all the formulas in $\Delta$ are true in the model (that is, take a value $\geqslant t$ ), and (4) the interpretation of $\epsilon^{t}=\delta^{t}$ is false in the model. Now assign values to the variables $X, Y, Z$ in the model in accordance with their definitions. Since $M(V)$ is a Boolean $t$-monoid, and the conditions of Lemmas 3 and 4 are satisfied, the complex expressions substituted for the variables in the formulas by the substitution $\sigma$ take the same values as the variables themselves. It follows that all of the formuls in $\Delta^{\sigma}$ are true in the model, but $\left(\epsilon^{t}=\delta^{t}\right)^{\sigma}$ is false in the model, showing that $\left(\epsilon^{t}=\delta^{t}\right)^{\sigma}$ is not deducible from $\Delta^{\sigma}$.

This proves part (I) of the theorem; part (2) follows exactly as in [8].

This seems an appropriate place to point out the error in the earlier attempt at a reduction to five variables. The passage describing this attempted reduction reads (in full) as follows:

In another direction, we can show that for extensions of $\mathbf{T}-\mathbf{W}+\mathbf{A r} 5$, the decision problem for formulas in five variables is unsolvable. Let $a_{1}, \ldots, a_{4}, c_{12}, \ldots, c_{34}$ be the 4 -frame in $M(V)$ in Lemma 4.6. Let $p=a_{1} \vee a_{2}, q=a_{1} \vee a_{3}, c=c_{12} \vee c_{13} \vee \ldots \vee c_{34}$. Then $p \wedge c=t$ so we can define $\sim x=\neg x \vee t$. Then elementary linear algebra shows that $a_{1}=p \wedge q, a_{2}=p \wedge \sim q, a_{3}=\sim p \wedge q$, $a_{4}=\sim p \wedge \sim q$; we can then define $c_{i j}=c \wedge\left(a_{i} \circ a_{j}\right)$. Since there is a finitely presented semigroup with two generators with unsolvable word problem, it follows that we can prove undecidability for 
formulas in five variables. A similar argument shows undecidability for formulas in six variables for the positive systems [8, p. I070].

Unfortunately for this argument, elementary linear algebra shows nothing of the sort. The trouble lies with the equation $a_{4}=\sim p \wedge \sim q$. Let us recall that the model $M(V)$ is constructed from an infinite-dimensional vector space $V$ with algebraic basis $\left\{e_{i}: i \in N\right\}=E$ (for details, see Lipshitz [2] ). The element $a_{i}$ is the subspace generated by the set $E_{i}=\left\{e_{i+4 k}: k \in N\right\}$. Now consider the vector $e_{1}+e_{2}$. This is not in the subspaces generated by $E_{1}, E_{2}$ or $E_{3}$, hence $e_{1}+e_{2} \in \sim p \wedge \sim q$. However, it is clear that $e_{1}+e_{2} \notin a_{4}$, so the purported equality is in fact false. For those who are curious about the source of this error, it resulted from confusing the Boolean complement in $M(V)$ with the complementation operation defined on the elements of the coordinate frame generated by $\left\{a_{1}, \ldots, a_{4}\right\}$.

\section{FURTHER PROBLEMS}

It is quite probable that the techniques of this paper can be pushed somewhat further. With sufficient ingenuity they can very likely be made to yield undecidability in three variables. I worked for a while on this, but eventually gave up, partly because I was tired, and partly because I liked the title, emulating the proud announcement "Four colors suffice" that the University of Illinois Mathematics Department added to its postage stamps when the Four Color Conjecture became the Four Color Theorem. Logicians who would like to work along this direction should note that the problem is made easier by the following fact. In trying to generate the frame and the two elements $r, s$ from a set of three rather than four elements, it is all right to assume that you are working in the model $M(V)$ (a much stronger assumption than the assumption of Lemmas 4 and 5). The model $M(V)$ has a very rich geometrical structure, and it may be possible to exploit this structure to cut down further on the number of variables.

I am inclined to conjecture that the two variable fragment is undecidable, though I doubt whether this can be proved using the geometrical techniques of $[8]$. Perhaps some new idea for proving undecidability is required. Undecidability in two variables, at least for the logic $\mathbf{R}$, would follow immediately if there were a freely associative connective in $\mathbf{R}$ in the sense of Thistlewaite, McRobbie and Meyer [6. However, after some efforts looking for such a connective, I have come to doubt whether there is such a thing, so this approach may be a dead end. The problem of whether such a connective exists still seems to me intriguing. You can prove the existence of a freely associative connective by exhibiting it. But how do you prove its non-existence?

The one-variable fragment is a mystery, unlike the zero-variable fragment. I venture the conjecture that it is decidable. But who knows? 


\section{REFERENCES}

[r] A. R. Anderson, N. D. Belnap Jr., and J. M. Dunn. Entailment, volume 2. Princeton University Press, Princeton, NJ, 1992.

[2] L. Lipshitz. The undecidability of the word problems for projective geometries and modular lattices. Transactions of the American Mathematical Society, I93:I7I-I80, I974.

[3] R.K. Meyer. Career induction ends here and here $=2$. Fournal of Philosophical Logic, pages 36I-I7I, 1979.

[4] Jörg Siekmann and Graham Wrightson, editors. Automation of Reasoning. Springer-Verlag, New York, 1983.

[5] John K. Slaney. 3088 varieties: A solution to the Ackermann constant problem. Fournal of Symbolic Logic, pages 487-501, 1985 .

[6] P.B. Thistlewaite, M.A. McRobbie, and R.K. Meyer. Automated theoremproving in non-classical logics. Pitman, London, 1988.

[7] G.S. Tseitin. On the complexity of derivation in propositional calculus. In A. O. Slisenko, editor, Studies in Constructive Mathematics and Mathematical Logic, Part 2, pages II5-I25. Consultants Bureau, New York, 1970. Reprinted in [4], Vol. 2, pp. 466-483.

[8] Alasdair Urquhart. The undecidability of entailment and relevant implication. Fournal of Symbolic Logic, 49:1059-1073, 1984. 
The Australasian fournal of Logic (ISSN I448-5052) disseminates articles that significantly advance the study of logic, in its mathematical, philosophical or computational guises. The scope of the journal includes all areas of logic, both pure and applied to topics in philosophy, mathematics, computation, linguistics and the other sciences.

Articles appearing in the journal have been carefully and critically refereed under the responsibility of members of the Editorial Board. Only papers judged to be both significant and excellent are accepted for publication.

The journal is freely available at the journal website at

$$
\text { http://www.philosophy.unimelb.edu.au/ajl/ }
$$

All issues of the journal are archived electronically at the journal website.

SuBSCRIPTIONS Individuals may subscribe to the journal by sending an email, including a full name, an institutional affiliation and an email address to the managing editor at ajl-editors@unimelb.edu.au Subscribers will receive email abstracts of accepted papers to an address of their choice. For institutional subscription, please email the managing editor at ajl-editors@unimelb.edu.au.

Complete published papers may be downloaded at the journal's website at http: //www.philosophy.unimelb.edu.au/ajl/ The journal currently publishes in pdf format.

Submission The journal accepts submissions of papers electronically. To submit an article for publication, send the $\mathrm{LT}_{\mathrm{E}} \mathrm{X}$ source of a submission to a member of the editorial board. For a current list of the editorial board, consult the website.

The copyright of each article remains with the author or authors of that article. 